\title{
HOW THE $\mu$-DEFORMED SEGAL-BARGMANN SPACE GETS TWO MEASURES
}

\author{
STEPHEN BRUCE SONTZ \\ Centro de Investigación en Matemáticas, A.C. (CIMAT) \\ Guanajuato, Mexico \\ E-mail:sontz@cimat.mx
}

\begin{abstract}
This note explains how the two measures used to define the $\mu$-deformed SegalBargmann space are natural and essentially unique structures. As is well known, the density with respect to Lebesgue measure of each of these measures involves a Macdonald function. Our primary result is that these densities are the solution of a system of ordinary differential equations which is naturally associated with this theory. We then solve this system and find the known densities as well as a "spurious" solution which only leads to a trivial holomorphic Hilbert space. This explains how the Macdonald functions arise in this theory. Also we comment on why it is plausible that only one measure will not work. We follow Bargmann's approach by imposing a condition sufficient for the $\mu$-deformed creation and annihilation operators to be adjoints of each other. While this note uses elementary techniques, it reveals in a new way basic aspects of the structure of the $\mu$-deformed Segal-Bargmann space.
\end{abstract}

1. Introduction. Before getting into details, let us remark that we will be studying deformations, depending on a dimensionless deformation parameter $\mu>-1 / 2$ (which is fixed throughout the note), of standard analysis and quantum mechanics. Our goal is to provide motivation for Definitions 1.1 and 1.2 below, including an explanation of the appearance of the Macdonald function in those definitions.

We start by recalling some definitions and notation that appear in [3]. Also see [2], [12], 13], 14] and [18] and references therein for other related work.

Definition 1.1. Say $\lambda>0$ We define measures on the complex plane $\mathbf{C}$ by

$$
\begin{aligned}
& d \nu_{e, \mu, \lambda}(z):=\nu_{e, \mu, \lambda}(z) d x d y, \\
& d \nu_{o, \mu, \lambda}(z):=\nu_{o, \mu, \lambda}(z) d x d y,
\end{aligned}
$$

2000 Mathematics Subject Classification: 46E20, 81S99.

Key words and phrases: Segal-Bargmann analysis, $\mu$-deformed quantum mechanics.

The paper is in final form and no version of it will be published elsewhere. 
whose densities are defined by

$$
\begin{gathered}
\nu_{e, \mu, \lambda}(z):=\lambda \frac{2^{\frac{1}{2}-\mu}}{\pi \Gamma\left(\mu+\frac{1}{2}\right)} K_{\mu-\frac{1}{2}}\left(\left|\lambda^{\frac{1}{2}} z\right|^{2}\right)\left|\lambda^{\frac{1}{2}} z\right|^{2 \mu+1} \\
\nu_{o, \mu, \lambda}(z):=\lambda \frac{2^{\frac{1}{2}-\mu}}{\pi \Gamma\left(\mu+\frac{1}{2}\right)} K_{\mu+\frac{1}{2}}\left(\left|\lambda^{\frac{1}{2}} z\right|^{2}\right)\left|\lambda^{\frac{1}{2}} z\right|^{2 \mu+1}
\end{gathered}
$$

for $0 \neq z \in \mathbf{C}$, where $\Gamma$ (the Euler gamma function) and $K_{\alpha}$ (the Macdonald function of order $\alpha$ ) are defined in [10]. Moreover, $d x d y$ is Lebesgue measure on $\mathbf{C}$.

The function $K_{\alpha}$ is also known as the modified Bessel function of the third kind or Basset's function. (See [7, p. 5.) But it is also simply known as a modified Bessel function. (See [8], p. 961, and [1], p. 374.) An explanation of where the Macdonald functions in Definition 1.1 come from was the motivation for writing this note.

From the formulas 1.1 and 1.2 , one can see why the case $\mu=-1 / 2$ has not been included. One should refer to the discussion of the Bose-like oscillator in [15] (especially, note Theorem 5.7) for motivation for the condition $\mu>-1 / 2$.

Let $\mathcal{H}(\mathbf{C})$ be the space of all holomorphic functions $f: \mathbf{C} \rightarrow \mathbf{C}$. We note that $f_{e}:=(f+J f) / 2$ (respectively, $f_{o}:=(f-J f) / 2$ ) defines the even (respectively, odd) part of $f$, where $J f(z):=f(-z)$ for all $z \in \mathbf{C}$ is the parity operator. So, $f=f_{e}+f_{o}$.

We use throughout the article the standard notations for $L^{2}$ spaces, for their inner products, and for their norms.

Definition 1.2. The $\mu$-deformed Segal-Bargmann space for $\lambda>0$ is

$$
\mathcal{B}_{\mu, 1 / \lambda}^{2}:=\mathcal{H}(\mathbf{C}) \cap\left\{f: \mathbf{C} \rightarrow \mathbf{C} \mid f_{e} \in L^{2}\left(\mathbf{C}, \nu_{e, \mu, \lambda}\right) \text { and } f_{o} \in L^{2}\left(\mathbf{C}, \nu_{o, \mu, \lambda}\right)\right\},
$$

where $f=f_{e}+f_{o}$ is the decomposition of a function into its even and odd parts. Next we define the norm

$$
\|f\|_{\mathcal{B}_{\mu, 1 / \lambda}^{2}}:=\left(\left\|f_{e}\right\|_{L^{2}\left(\mathbf{C}, \nu_{e, \mu, \lambda}\right)}^{2}+\left\|f_{o}\right\|_{L^{2}\left(\mathbf{C}, \nu_{o, \mu, \lambda}\right)}^{2}\right)^{1 / 2}
$$

for all $f \in \mathcal{B}_{\mu, 1 / \lambda}^{2}$.

This definition is due to Marron in [11] and Rosenblum in [16. The reason for using $1 / \lambda$ instead of $\lambda$ in the notation has to do with maintaining consistency with the notation of Hall in [9]. For more on the historical background of this definition, see [3]. We have that $\mathcal{B}_{\mu, 1 / \lambda}^{2}$ is a Hilbert space (see [1]) with inner product defined by

$$
\langle f, g\rangle_{\mathcal{B}_{\mu, 1 / \lambda}^{2}}:=\left\langle f_{e}, g_{e}\right\rangle_{L^{2}\left(\nu_{e, \mu, \lambda}\right)}+\left\langle f_{o}, g_{o}\right\rangle_{L^{2}\left(\nu_{o, \mu, \lambda}\right)}
$$

Of course, $f=f_{e}+f_{o}$ and $g=g_{e}+g_{o}$ are the representations of $f$ and $g$ as the sums of their even and odd parts. (We will often use such representations without explicit comment, letting the notation carry the burden of explanation.) When $\mu=0$ and $\lambda=1$ this reduces to the usual Segal-Bargmann space, denoted here by $\mathcal{B}^{2}$. (See [5, 17].) For simplicity of notation we put $\lambda=1$ for the rest of this article. We also put $\mathcal{B}_{\mu}^{2}=\mathcal{B}_{\mu, 1}^{2}$

There is a way of seeing a relation of this article to standard Segal-Bargmann analysis. In general this is to find relations in the $\mu$-deformed theory that do not depend on the parameter $\mu$. This means that the relations for $\mu \neq 0$ are exactly the same as those for the standard case $\mu=0$. This will be our approach in Section 2 where we motivate the 
definition of the measures in the $\mu$-deformed Segal-Bargmann space and, in particular, show how the Macdonald functions arise naturally.

2. The measures in the Segal-Bargmann spaces. It turns out that $\mathcal{B}_{\mu}^{2}$ is the image of a $\mu$-deformed Segal-Bargmann transform (see [11]) which is unitary. This unitarity is a consequence of the definition of the inner product on $\mathcal{B}_{\mu}^{2}$ in terms of the two measures defined in Definition 1.1. We wish to motivate this definition of the inner product intrinsically, that is, without reference to the $\mu$-deformed Segal-Bargmann transform but rather as a basic structure that arises naturally for holomorphic functions. We will do this modulo a normalization factor that is left undetermined intrinsically. Rather than prove theorems, the purpose of this note is to show how the definitions $[1.1$ and 1.2 are naturally motivated. We follow an idea given in [5]. To achieve this we will use $\mu$-deformed creation and annihilation operators defined for arbitrary holomorphic functions.

Definition 2.1. Let $f \in \mathcal{H}(\mathbf{C})$ be a holomorphic function and $z \in \mathbf{C}$. Then the $\mu$ deformed creation operator $a_{\mu}^{*}: \mathcal{H}(\mathbf{C}) \rightarrow \mathcal{H}(\mathbf{C})$ is defined by

$$
a_{\mu}^{*} f(z):=z f(z) .
$$

The $\mu$-deformed annihilation operator $a_{\mu}: \mathcal{H}(\mathbf{C}) \rightarrow \mathcal{H}(\mathbf{C})$ is defined by

$$
a_{\mu} f(z):=\frac{\partial f}{\partial z}+\frac{\mu}{z}(f(z)-f(-z)),
$$

(Here we use the standard notation $\partial / \partial z=(1 / 2)(\partial / \partial x-i \partial / \partial y)$ from complex variable theory for the complex derivative operator. We also mention in passing that $a_{\mu}$ is a variant of the Dunkl operator adequate for complex variable theory.)

These operators satisfy the $\mu$-deformed commutation relation

$$
\left[a_{\mu}, a_{\mu}^{*}\right]=I+2 \mu J
$$

on $\mathcal{H}(\mathbf{C})$. This commutation relation, which is the central identity of $\mu$-deformed quantum mechanics, was originally introduced by Wigner in [19].

For example, in the original theory in [5] when $\mu=0$, Bargmann defines the inner product in terms of a measure on the phase space, namely,

$$
\langle f, g\rangle_{\mathcal{B}_{0}^{2}}:=\int_{\mathbf{C}} d x d y \nu_{\text {Gauss }}(z) f(z)^{*} g(z)
$$

for all holomorphic functions $f$ and $g$ that are in $L^{2}\left(\mathbf{C}, \nu_{\text {Gauss }}\right)$. Here, the density $\nu_{\text {Gauss }}$ is defined by

$$
\nu_{\text {Gauss }}(z):=\frac{1}{\pi} e^{-|z|^{2}}
$$

for all $z \in \mathbf{C}$. Moreover, Bargmann's motivation for this definition of the measure on the phase space comes from formally analyzing for holomorphic $f$ and $g$ the condition

$$
\left\langle a_{0}^{*} f, g\right\rangle_{L^{2}(\mathbf{C}, \nu)}=\left\langle f, a_{0} g\right\rangle_{L^{2}(\mathbf{C}, \nu)},
$$

where $\nu(z)$ is an unknown density function which defines the measure $\nu(z) d x d y$ on $\mathbf{C}$. We can try a similar strategy for the $\mu$-deformed creation and annihilation operators $a_{\mu}^{*}$ 
and $a_{\mu}$ in place of the usual creation and annihilation operators $a_{0}^{*}$ and $a_{0}$. So we want to consider, again for $f$ and $g$ holomorphic, the identity

$$
\left\langle a_{\mu}^{*} f, g\right\rangle_{L^{2}\left(\mathbf{C}, \nu_{\mu}\right)}=\left\langle f, a_{\mu} g\right\rangle_{L^{2}\left(\mathbf{C}, \nu_{\mu}\right)}
$$

and try to find the unknown density function $\nu_{\mu}(z)$ for a measure $\nu_{\mu}(z) d x d y$ on $\mathbf{C}$. We have been unable to prove that this has no solution when $\mu \neq 0$, though this seems to be the case. However, we have shown that the sufficient condition on $\nu_{\mu}(z)$ given by a formal integration by parts argument (as done by Bargmann in [5]) has no solution if $\mu \neq 0$. We will come back to this point later.

In any event, what Marron in [1] and Rosenblum in [16] did was to define two measures on the phase space $\mathbf{C}$ and use formula $(1.3)$ to define the inner product. We do not know what motivation they had to write down these measures, but we have been able to construct the following intuitive reasoning à la Bargmann in [5]. We do not believe that the following exposition is new. Indeed, we fully expect it was known to Rosenblum. However, we have not found it in the literature. Unfortunately, Marvin Rosenblum died some time after giving us a copy of [16], which is a sketchy preliminary document that was as far as we know never put into a publishable form.

First, we consider the desired relation

$$
\left\langle a_{\mu}^{*} f, g\right\rangle_{\mathcal{B}_{\mu}^{2}}=\left\langle f, a_{\mu} g\right\rangle_{\mathcal{B}_{\mu}^{2}} .
$$

Since the non-local parity operator $J$ figures in the $\mu$-deformed canonical commutation relation (2.1), it seems plausible to divide $\mathcal{H}(\mathbf{C})$ into the two eigenspaces for this operator, i.e., the subspaces of even and odd functions, respectively. So we propose to introduce two measures with densities $\nu_{e}$ and $\nu_{o}$ on the phase space $\mathbf{C}$ and define an inner product by

$$
\langle f, g\rangle_{\mathcal{B}_{\mu}^{2}}:=\left\langle f_{e}, g_{e}\right\rangle_{L^{2}\left(\nu_{e}\right)}+\left\langle f_{o}, g_{o}\right\rangle_{L^{2}\left(\nu_{o}\right)},
$$

using the even and odd parts of $f$ and $g$ on the right hand side of this definition.

Next, we want to see what restriction 2.3 places on the unknowns $\nu_{e}$ and $\nu_{o}$. Since $a_{\mu}^{*}$ and $a_{\mu}$ interchange the even and odd subspaces of $\mathcal{H}(\mathbf{C})$ and since these are orthogonal subspaces for the proposed inner product 2.4, there are exactly two non-trivial cases of 2.3. The first such case is for $f$ even and $g$ odd. Then we have the condition

$$
\left\langle a_{\mu}^{*} f, g\right\rangle_{L^{2}\left(\nu_{o}\right)}=\left\langle f, a_{\mu} g\right\rangle_{L^{2}\left(\nu_{e}\right)} .
$$

The second non-trivial case is for $f$ odd and $g$ even, in which case we have

$$
\left\langle a_{\mu}^{*} f, g\right\rangle_{L^{2}\left(\nu_{e}\right)}=\left\langle f, a_{\mu} g\right\rangle_{L^{2}\left(\nu_{o}\right)} .
$$

Next, we write out these conditions in terms of integrals. The first condition 2.5 gives

$$
\begin{gathered}
\int_{\mathbf{C}} d x d y \nu_{o}(z) z^{*} f(z)^{*} g(z)=\int_{\mathbf{C}} d x d y \nu_{e}(z) f(z)^{*} a_{\mu} g(z) \\
=\int_{\mathbf{C}} d x d y \nu_{e}(z) f(z)^{*}\left(\frac{\partial g}{\partial z}+\frac{2 \mu}{z} g(z)\right) \\
=\int_{\mathbf{C}} d x d y\left(-\frac{\partial \nu_{e}}{\partial z}+\nu_{e}(z) \frac{2 \mu}{z}\right) f(z)^{*} g(z)
\end{gathered}
$$


where we used the fact that $g$ is odd to calculate $a_{\mu} g$ and then we integrated formally by parts, using $\partial f^{*} / \partial z=0$ since $f$ is holomorphic.

The second condition 2.6 gives

$$
\begin{gathered}
\int_{\mathbf{C}} d x d y \nu_{e}(z) z^{*} f(z)^{*} g(z)=\int_{\mathbf{C}} d x d y \nu_{o}(z) f(z)^{*} a_{\mu} g(z) \\
=\int_{\mathbf{C}} d x d y \nu_{o}(z) f(z)^{*} \frac{\partial g}{\partial z} \\
=\int_{\mathbf{C}} d x d y\left(-\frac{\partial \nu_{o}}{\partial z}\right) f(z)^{*} g(z),
\end{gathered}
$$

where we used the fact that $g$ is even to evaluate $a_{\mu} g$. Again, the last equality is a formal integration by parts. Clearly a sufficient condition for these two conditions (2.7) and (2.8) to hold is this system:

$$
\begin{gathered}
z^{*} \nu_{o}(z)=-\frac{\partial \nu_{e}}{\partial z}+\nu_{e}(z) \frac{2 \mu}{z} \\
z^{*} \nu_{e}(z)=-\frac{\partial \nu_{o}}{\partial z}
\end{gathered}
$$

which is equivalent to

$$
\begin{gathered}
|z|^{2} \nu_{o}(z)=-z \frac{\partial \nu_{e}}{\partial z}+2 \mu \nu_{e}(z), \\
|z|^{2} \nu_{e}(z)=-z \frac{\partial \nu_{o}}{\partial z} .
\end{gathered}
$$

While this is a sufficient condition for $(2.5)$ and $(2.6)$, it is not clear whether it is also necessary, since the functions $f$ and $g$ in the integral identities 2.5 and $(2.6)$ are both holomorphic and of specific parities.

If we try to use only one measure on $\mathbf{C}$ and impose Bargmann's condition, we find (by almost the same argument) that $(2.9)$ and $(2.10)$ hold provided that we set $\nu_{e}=\nu_{o}$ in them. But this pair of equations would then have no nonzero solution for $\mu \neq 0$. This leads us to believe that the $\mu$-deformed Segal-Bargmann space cannot be realized as a subspace of $L^{2}(\mathbf{C}, \nu)$ for some measure $\nu$ that is absolutely continuous with respect to Lebesgue measure.

Next we note that in the standard polar coordinates $r, \theta$ in $\mathbf{C}$ we have that

$$
z \frac{\partial}{\partial z}=\frac{1}{2}\left(r \frac{\partial}{\partial r}-i \frac{\partial}{\partial \theta}\right) .
$$

Since we are seeking real solutions $\nu_{e}$ and $\nu_{o}$, it follows by equating imaginary (respectively, real) parts on both sides of 2.9 and (2.10, when written in polar coordinates, that

$$
\begin{aligned}
\frac{\partial}{\partial \theta} \nu_{e} & =0, & r^{2} \nu_{o} & =-\frac{1}{2} r \frac{\partial}{\partial r} \nu_{e}+2 \mu \nu_{e}, \\
\frac{\partial}{\partial \theta} \nu_{o} & =0, & r^{2} \nu_{e} & =-\frac{1}{2} r \frac{\partial}{\partial r} \nu_{o} .
\end{aligned}
$$

Thus, we are looking for two functions of $r>0$ only (since they do not depend on $\theta$ according to the first two equations) satisfying these last two equations, which form a first 
order, homogeneous linear system with variable real-valued coefficients in the unknown pair $\left(\nu_{e}, \nu_{o}\right)$. Therefore these real-valued solutions satisfy the coupled equations

$$
\begin{gathered}
\nu_{o}=-\frac{1}{2 r} \frac{d}{d r} \nu_{e}+\frac{2 \mu}{r^{2}} \nu_{e}, \\
\nu_{e}=-\frac{1}{2 r} \frac{d}{d r} \nu_{o},
\end{gathered}
$$

and so form a vector space of dimension two over $\mathbf{R}$. Substituting the first equation into the second, we get

$$
\frac{d^{2} \nu_{e}}{d r^{2}}-\frac{(1+4 \mu)}{r} \frac{d \nu_{e}}{d r}+\left(\frac{8 \mu}{r^{2}}-4 r^{2}\right) \nu_{e}=0,
$$

while by substituting the second into the first, we have that

$$
\frac{d^{2} \nu_{o}}{d r^{2}}-\frac{(1+4 \mu)}{r} \frac{d \nu_{o}}{d r}-4 r^{2} \nu_{o}=0 .
$$

Now 2.13 and (2.14) are decoupled second order linear differential equations, which impose sufficient conditions on the solutions $\nu_{e}$ and $\nu_{o}$ of the original coupled system 2.11 and 2.12). Since each one of the equations 2.13 and 2.14 has a space of realvalued solutions of dimension two over $\mathbf{R}$, the pairs of these solutions $\left(\nu_{e}, \nu_{o}\right)$ form a space of dimension four, which includes the two dimensional space of solutions of the coupled system 2.11 and 2.12). Our method will be to find the general solution of both of the equations (2.13) and (2.14) and then identify the two dimensional subspace of solutions to the coupled system.

Making the change of dependent variable, $\nu_{e}(r)=r^{\alpha} \phi\left(r^{2}\right)$ in 2.13 we find that

$$
\phi^{\prime \prime}\left(r^{2}\right)+\frac{\alpha-2 \mu}{r^{2}} \phi^{\prime}\left(r^{2}\right)+\left(\frac{\alpha^{2}-2 \alpha-4 \alpha \mu+8 \mu}{4 r^{4}}-1\right) \phi\left(r^{2}\right)=0,
$$

which looks something like Bessel's equation of order $\nu$ ([10], p. 98), namely

$$
u^{\prime \prime}(x)+\frac{1}{x} u^{\prime}(x)+\left(1-\frac{\nu^{2}}{x^{2}}\right) u(x)=0
$$

for $x=r^{2}$. (This change of variable may seem unmotivated, as is often the case with this method, but it is really not that unusual.) To get better agreement with the form of Bessel's equation, we choose the exponent $\alpha$ in the change of variable such that $\alpha-2 \mu=1$, that is $\alpha=2 \mu+1$. With this value for $\alpha$ we calculate that $\alpha^{2}-2 \alpha-4 \alpha \mu+8 \mu=-4(\mu-1 / 2)^{2}$ and so 2.15 becomes

$$
\phi^{\prime \prime}\left(r^{2}\right)+\frac{1}{r^{2}} \phi^{\prime}\left(r^{2}\right)-\left(\frac{(\mu-1 / 2)^{2}}{r^{4}}+1\right) \phi\left(r^{2}\right)=0,
$$

which is not Bessel's equation but rather a related equation known as Bessel's modified equation of order $\nu$, namely

$$
u^{\prime \prime}(x)+\frac{1}{x} u^{\prime}(x)-\left(1+\frac{\nu^{2}}{x^{2}}\right) u(x)=0
$$

with $x=r^{2}$ and $\nu=\mu-1 / 2$. Its general real valued solution $([10$, p. 110) is

$$
\phi\left(r^{2}\right)=a I_{\mu-1 / 2}\left(r^{2}\right)+b K_{\mu-1 / 2}\left(r^{2}\right),
$$


where $I_{\mu-1 / 2}$ is the modified Bessel function of the first kind and of order $\mu-1 / 2, K_{\mu-1 / 2}$ is the Macdonald function of order $\mu-1 / 2$ and $a, b \in \mathbf{R}$. See [10] for more details about these special functions. Consequently,

$$
\nu_{e}(r)=r^{2 \mu+1}\left[a I_{\mu-1 / 2}\left(r^{2}\right)+b K_{\mu-1 / 2}\left(r^{2}\right)\right]
$$

is the general real valued solution of 2.13 , where $a, b \in \mathbf{R}$.

Similarly the change of variable, $\nu_{o}(r)=r^{\alpha} \psi\left(r^{2}\right)$ converts 2.14 into

$$
\psi^{\prime \prime}\left(r^{2}\right)+\frac{\alpha-2 \mu}{r^{2}} \psi^{\prime}\left(r^{2}\right)+\left(\frac{\alpha^{2}-2 \alpha-4 \alpha \mu}{4 r^{4}}-1\right) \psi\left(r^{2}\right)=0,
$$

and again we choose $\alpha=2 \mu+1$ for the same reason as before. So, $\alpha^{2}-2 \alpha-4 \alpha \mu=$ $-4(\mu+1 / 2)^{2}$ follows and (2.17) becomes

$$
\psi^{\prime \prime}\left(r^{2}\right)+\frac{1}{r^{2}} \psi^{\prime}\left(r^{2}\right)-\left(\frac{(\mu+1 / 2)^{2}}{r^{4}}+1\right) \psi\left(r^{2}\right)=0 .
$$

This is again Bessel's modified equation, but now of order $\mu+1 / 2$ instead of $\mu-1 / 2$. So its general real valued solution is

$$
\psi\left(r^{2}\right)=c I_{\mu+1 / 2}\left(r^{2}\right)+d K_{\mu+1 / 2}\left(r^{2}\right),
$$

where $c, d \in \mathbf{R}$. Thus

$$
\nu_{o}(r)=r^{2 \mu+1}\left[c I_{\mu+1 / 2}\left(r^{2}\right)+d K_{\mu+1 / 2}\left(r^{2}\right)\right]
$$

is the general real valued solution of 2.14. It only remains to eliminate the superfluous solutions, namely the solutions of the individually decoupled equations that do not pair up to give a solution of the coupled system 2.11 and 2.12. For example, starting with the right side of 2.12 and putting $\nu_{o}(r)=r^{2 \mu+1} K_{\mu+1 / 2}\left(r^{2}\right)$ and using $s=r^{2}$ we see that

$$
\begin{gathered}
\left(-\frac{1}{2 r}\right) \frac{d}{d r}\left(r^{2 \mu+1} K_{\mu+1 / 2}\left(r^{2}\right)\right)=-\frac{d}{d s}\left(s^{\mu+1 / 2} K_{\mu+1 / 2}(s)\right) \\
=s^{\mu+1 / 2} K_{\mu-1 / 2}(s)=r^{2 \mu+1} K_{\mu-1 / 2}\left(r^{2}\right)
\end{gathered}
$$

where the second equality is an identity that can be found in [10], p. 110. This shows that the pair

$$
\left(r^{2 \mu+1} K_{\mu-1 / 2}\left(r^{2}\right), r^{2 \mu+1} K_{\mu+1 / 2}\left(r^{2}\right)\right)
$$

is a solution of the coupled system. Since $K_{-1 / 2}(z)=K_{1 / 2}(z)$ (see [10]), we see that these two densities are equal when $\mu=0$. But when $\mu \neq 0$, these densities are not equal and so we find that our sufficient condition does not give one measure on the phase space, but rather two.

Since the coupled system solution space has dimension two, we still need one more linearly independent pair solving the coupled system. But we have the following calculation that is very similar to the previous one:

$$
\begin{gathered}
\left(-\frac{1}{2 r}\right) \frac{d}{d r}\left(r^{2 \mu+1} I_{\mu+1 / 2}\left(r^{2}\right)\right)=-\frac{d}{d s}\left(s^{\mu+1 / 2} I_{\mu+1 / 2}(s)\right) \\
=-s^{\mu+1 / 2} I_{\mu-1 / 2}(s)=-r^{2 \mu+1} I_{\mu-1 / 2}\left(r^{2}\right)
\end{gathered}
$$


by another identity from [10, p. 110. So the pair

$$
\left(-r^{2 \mu+1} I_{\mu-1 / 2}\left(r^{2}\right), r^{2 \mu+1} I_{\mu+1 / 2}\left(r^{2}\right)\right)
$$

is another, linearly independent solution of the coupled system. Now, $K_{\nu}(x)>0$ and $I_{\nu}(x)>0$ for all $x>0$. This means that the solution 2.19 gives a pair of positive densities, which then define positive measures. However, the solution 2.20) gives a pair of densities with opposite signs even after multiplying by any nonzero real number. This in itself is not a fatal flaw with the solution 2.20 since one can develop interesting theories with signed measures. The real problem with this solution is its asymptotic growth as $r \rightarrow \infty$ for each function in the pair. (Precise information on this growth to infinity can be found in [10] but that is not relevant to this discussion.) Since we are looking for measures for holomorphic functions which, except for the constants, also go to infinity as $r \rightarrow \infty$, there is no way to use the solution 22.20) to construct a pair of nontrivial holomorphic $L^{2}$ function spaces. On the other hand, both of the functions in 2.19 are integrable with respect to the measure $r d r$, which is the radial part of Lebesgue measure $r d r d \theta$ in polar coordinates. (See [3] or [13].) With a suitable normalization either one of the functions in 2.19 (but not both if $\mu \neq 0$ ) can be made into a probability measure. The definition in 1.1 and 1.2 has the normalization that makes $d \nu_{e, \mu}$ into a probability measure. (Recall again that we are only considering in detail here the case $\lambda=1$, but the case for general $\lambda>0$ follows immediately.)

We can understand the particular normalization in $\sqrt{1.1}$ and $\sqrt{1.2}$ in terms of the $\mu$ deformed Segal-Bargmann transform $B_{\mu}$. (This paragraph uses definitions and notations from [18, which should be consulted for more details.) It turns out that $B_{\mu}$ has been normalized so that $B_{\mu} 1=1$. But 1 is a unit vector in the domain $L^{2}\left(\mathbf{R}, d \rho_{\mu}\right)$ of $B_{\mu}$, since $d \rho_{\mu}$ is a probability measure. Since we want $B_{\mu}$ to be a unitary transform, a necessary condition is that 1 in the codomain also be a unit vector. Since 1 is an even function, this forces $d \nu_{e, \mu}$ to be a probability measure, while imposing no restriction on $d \nu_{o, \mu}$. Clearly the normalization of the two measures cannot be made intrinsic to the holomorphic side of the theory by just using the Bargmann condition, which is itself homogeneous in the measures.

We would like to close this presentation of our understanding of where the Macdonald functions in the $\mu$-deformed Segal-Bargmann space come from by reiterating that all of this discussion fits into the way of understanding why this theory should be thought of as a type of Segal-Bargmann analysis, as we described at the very end of Section 1 . This is because the relation between the creation and annihilation operators, namely that they are adjoints, does not depend on the deformation parameter $\mu$.

3. Open problems. A completely different way was introduced by Hall in [9] for defining an intrinsic inner product on the codomain of his generalized Segal-Bargmann transforms. This is done in terms of a heat kernel measure defined on the phase space. We do not go into details here, but merely note that his method produces only one measure (for each of his three versions: $A, B$ and $C$ ), and so it appears not to be applicable to the case of the $\mu$-deformed Segal-Bargmann transform, where we have two measures when $\mu \neq 0$. Notice that this relates to the general problem posed in [6] of finding some measure 
$\nu$ on the phase space in order to realize an abstract Hilbert space, introduced there as a generalized Segal-Bargmann space associated with a Coxeter group, as a subspace of $L^{2}(\nu)$. In particular, it indicates that the problem may be to find a finite family of measures on the phase space instead of merely one. It would be interesting if one could find a construction of the measures for the $\mu$-deformed Segal-Bargmann space using methods from heat kernel analysis, although this cannot be in strict analogy with Hall's method as we have noted above. We consider this is to be a major challenge remaining in this area of research.

Nothing in our discussion excludes the possibility that there may well be ways of putting three or more measures on the phase space and using them to construct a SegalBargmann type space together with an associated Segal-Bargmann transform. And we have not proved definitively that this theory cannot be made to work with only one measure, though this seems plausible on account of our earlier remarks. In this context, we should note that Asai has shown in [4] under some rather stringent hypotheses that the Segal-Bargmann space associated to a probability measure on the configuration space $\mathbf{R}$ can be realized as the $L^{2}$ space of holomorphic functions on the phase space $\mathbf{C}$ for a unique probability measure on $\mathbf{C}$. However, the case of $\mu$-deformed quantum mechanics considered here is not included among the cases considered in [4].

Finally, the "spurious" solution which we have found for the coupled system (2.11) and 2.12 may still be useful in the construction of some sort of related theory. This is a highly speculative as well as vague comment.

Acknowledgments. This note was begun during an academic visit at the Department of Mathematics of the University of Virginia during my sabbatical year in 2007. I wish to express my thanks to Larry Thomas, my host there. I also wish to thank N. Asai for bringing his article [4] to my attention. And I thank all of the organizers of the 11th Workshop on Non-harmonic Analysis held in Będlewo, Poland, during the week of 18-23 August 2008 for their cordial invitation to participate in that intellectually interesting, and challenging, event.

This research was partially supported by CONACYT (Mexico), project 49187.

\section{References}

[1] M. Abramowitz and I. A. Stegun, Handbook of Mathematical Functions, Dover, New York, 1965. (9th printing, 1972.)

[2] C. Angulo Aguila and S. B. Sontz, Reverse Inequalities in $\mu$-deformed Segal-Bargmann analysis, J. Math. Phys. 47 (2006), 042103, 21 pp.

[3] C. Angulo Aguila and S. B. Sontz, Direct and reverse log-Sobolev inequalities in $\mu$-deformed Segal-Bargmann analysis, Infin. Dimens. Anal. Quantum Probab. Relat. Top. 10 (2007), 539-571.

[4] N. Asai, Hilbert space of analytic functions associated with the modified Bessel function and related orthogonal polynomials, Inf. Dim. Anal. Q. Prob. Rel. Top. 8 (2005), 505-514.

[5] V. Bargmann, On a Hilbert space of analytic functions and an associated integral transform, Part I, Commun. Pure Appl. Math. 14 (1961), 187-214. 
[6] S. Ben Saïd and B. Ørsted, Segal-Bargmann transforms associated with finite Coxeter groups, Math. Ann. 334 (2006), 281-323.

[7] A. Erdélyi et al., Higher Transcendental Functions, Volume II, McGraw-Hill, New York, 1953. (Reprint edition, Krieger, Malabar, 1981.)

[8] I. S. Gradshteyn and I. M. Ryzhik, Tables of Integrals, Series and Products, 5th edition, Academic Press, San Diego, 1994.

[9] B. C. Hall, The Segal-Bargmann "coherent state" transform for compact Lie groups, J. Func. Anal. 122 (1994), 103-151.

[10] N. N. Lebedev, Special Functions and Their Applications, Dover, New York, 1972.

[11] C. S. Marron, Semigroups and the Bose-like oscillator, Ph. D. Dissertation, The University of Virginia, 1994.

[12] C. Pita and S. B. Sontz, On Hirschman and log-Sobolev inequalities in $\mu$-deformed SegalBargmann analysis, J. Phys. A: Math. Gen. 39 (2006), 8631-8662.

[13] C. Pita and S. B. Sontz, On Shannon entropies in $\mu$-deformed Segal-Bargmann analysis, J. Math. Phys. 47 (2006), 032101, 31 pp.

[14] C. Pita and S. B. Sontz, Accardi complementarity in $\mu$-deformed quantum mechanics, in: Proceedings of the XXIV Workshop in Geometric Methods in Physics (Białowieża, 2005), J. Geom. Symm. Phys. 6 (2006), 101-108.

[15] M. Rosenblum, Generalized Hermite polynomials and the Bose-like oscillator calculus, in: Nonselfadjoint Operators and Related Topics, Operator Theory Advances and Applications 73, A. Feintuch and I. Gohberg (eds.), Birkhäuser, 1994, 369-396.

[16] M. Rosenblum, Operators associated with the Bose-like oscillator, unpublished manuscript, 1994.

[17] I. E. Segal, Mathematical problems of relativistic physics, in: Proceedings of the Summer Seminar, Boulder, Colorado (1960), Vol. II, M. Kac (ed.), Lectures in Appl. Math., Am. Math. Soc., Providence, 1963.

[18] S. B. Sontz, The $\mu$-deformed Segal-Bargmann transform is a Hall type transform, Inf. Dim. Anal. Quantum Probab. Relat. Top. 12 (2009), 269-289.

[19] E. P. Wigner, Do the equations of motion determine the quantum mechanical commutation relations?, Phys. Rev. 77 (1950), 711-712. 